\title{
A Survey on Deep Learning Algorithms for Skin Lesion Classification
}

\author{
Merin Mathew ${ }^{1}$, Arulanand Natarajan ${ }^{2}$ \\ \{20mz32@psgtech.ac.in ${ }^{1}$, naa.cse@psgtech.ac.in $\left.{ }^{2}\right\}$ \\ PG Student, Department of CSE, PSG College Of Technology, Coimbatore, Tamil Nadu, India ${ }^{1}$, \\ Professor, Department of CSE, PSG College Of Technology, Coimbatore, Tamil Nadu, India ${ }^{2}$
}

\begin{abstract}
Skin cancer is a severe disease and is often ignored by the people at an early stage. Early detection of melanoma or other skin lesions with the help of dermoscopy images increases the survival rate.Analysis of skin lesion technique still experiences some challenges due to unique features of skin lesion images. This paper reviews the deep learning techniques that have been used for detecting different types of skin lesions. The topics include image preprocessing, image segmentation, feature extraction and classification on dermoscopy images of skin lesions. Deep learning techniques with well preprocessed and segmented lesion images shows better classification of skin lesion images.
\end{abstract}

Keywords: Deep Learning, Image Segmentation, Dermoscopy images, Preprocessing , Feature extraction, Skin lesion.

\section{Introduction}

Skin cancer is one of the major contributors to the cause of death all over the world. Skin lesions can be of different shape, color and size and can be either isolated or in groups. Skin lesion is an abnormal growth which appears in skin cells which generally develops in the skin areas that are exposed to the sun and can also form in places where the skin is not exposed to the sun . Skin Cancer occurs on the upper layer of the skin, epidermis, which can be seen by human eyes. Some skin cancer types are vascular lesion, squamous cell carcinoma, and melanoma. Melanoma is the deadliest skin cancer which occupies the nearby tissue and spreads to other parts of the body. Dermoscopy is one of the common imaging techniques used by dermatologists. Dermatoscopy is used to examine the skin condition using a skin surface microscopy.It evaluates the pigmented skin and is much easier to diagnose melanoma at its earlier stage by magnifying the surface of the skin lesion. Dermoscopicimages show both malignant or non-malignant lesions.

Deep learning techniques implement algorithms for learning purposes, which train the data and then test that data, by adopting their parameters. A complication occurs while evaluating the skin lesion classification models which uses imbalance of classes present in the databases making it difficult to classify and evaluate.

The deep convolutional neural networks and considering the excellent performance for image classification, and to utilise them for medical image analysis including skin lesion classification. 
Most of the skin cancer is diagnosed based on the ABCDE rule. Asymmetry, border irregularity, color of lesion, diameter of lesion and enlarging the lesion.

The commonly used dataset is ISIC 2019 which consist of 25,331 images containing images from eight classes used for training. Skin lesions in ISIC 2019 are classified into 8 groups: Melanoma, Vascular Lesion, Squamous Cell Carcinoma, Melanocytic Nevus,Basal Cell Carcinoma, Actinic Keratosis,Benign Keratosis,Dermatofibroma.

Basal cell carcinoma looks like a flesh colored bump, or pinkish patch in skin.Squamous cell carcinoma is a red firm bump with a scaly patch that heals and then reopens.Actinic keratoses are dry, scaly spots.Melanoma is developed in a mole or it appears suddenly as a new dark spot on the skin surface.Benign keratosis is a light brown which is round to oval and varies in size.Vascular lesions are bright red in color and grow frequently on top of the skin surface.

Dermatofibroma is a round bump mostly developed under the skin.Melanocytic nevus is spreading and changes in color of the cells of the skin.

The above 8 classes in dataset consist of 25,331 images Melanoma of 4522 ,Benign keratosis of 2624,Melanocytic nevus of 12,875,Dermatofibroma of 239, Basal cell carcinoma of 3323 ,Vascular lesion of 253,Actinic keratosis of 867and Squamous cell carcinoma of 628 dermoscopic images.

Image segmentation has made a tremendous progress in deep neural network where images are clustered together if it belongs to the same object class. This process is known as pixel-level classification. Multiple image segmentation techniques are developed based on graph cuts , conditional or sparsity based methods including the earlier methods like histogram-based bundling, k-means clustering or thresholding. Deep learning based segmentation model achieves the best accuracy on popular benchmarks.

In medical diagnosis of cancer, image segmentation technique called clustering is used to identify the tissue type present in the images. It divides the images into segments and only the important segments are processed instead of entire image.

The segmented images are then sent for feature extraction where the features from the segmented image are selected which is also a dimensionality reduction process. The features are extracted and selected using a ResNet, AlexNet or VGG16 algorithm .

Feature extraction helps to minimize the number of resource without losing any important information and also reduce the recurrent data for the analysis. Algorithms used for feature detection are based on the shape or edges in aimage. The number of feature maps present in each convolutional layer is the number of sampling layer. The features extracted are given to the classifier to classify the type of lesion.

\section{Literature review}

Initially, the major work was done for developing an automated model to diagnose lesions based on order irregularity, color of lesion, diameter of lesion and enlarging lesion properties. In these models $[5,8]$, they converted these properties of each image into numerical figures and generated a cumulative score by adding and diagnosing the images based on the cumulative score. 
Ali Madooei, et al, (2018) have developed anensemble deep learning methods which identifies the skin lesion from the dermoscopic images present in the ISIC 2018 dataset. It is designed by fusing intra architecture and interior architecture networks for the convolutional neural network. Therefore, accuracy for different studies is carried out by calculating the metrics such as precision, accuracy and the area under curve [8].

The efficiency is checked for model reliability and is used to classify the three classes in the dataset. The system is tested and trained on the PH2 dataset by achieving a high accuracy which brings down the credibility of the method by using only one limited dataset. Different techniques such as AlexNet and VGG16 are used on the $\mathrm{PH} 2$ dataset. $\mathrm{PH} 2$ dataset consists of only 200 images for testing and training. Due to less number of images present in the dataset leads to better performance, hence a larger dataset should be used to check the performance based on the above techniques. Only a few images of rare cancer are used to test and train . For obtaining better accuracy, the model should be tested on the multiple dataset to obtain an efficient result.

The system performs operation on skin lesions by categorizing the dataset to melanoma and benign where majority of finding is reviewed based on the analysis for binary classification of disease. The melanoma detection is done in five phases which includes data acquisition, fine tuning, feature extraction and selection, deep learning and model finalizing. Fine tuning is applied to the images were unnecessary regions such as wrinkles, hair were removed from the surface of the skin to obtain a better result .Feature extraction is an important phase where the features are extracted after the feature reduction technique which is applied to the bulk features . After the feature extraction and selection deep learning technique is used for the dataset to check the reliability of the methods.

The skin lesion analysis by ensemble the CNN methods using a ISIC 2019 dataset. The data have different resolution and class imbalance, so each image are resized using data augmentation technique. The images present in the dataset are segmented using R2U-Net which is a combination of U-net and ResNet . It eliminates the black area and then it is fed into the convolutional network.

After the removal of the dark spots in the images it is given for the feature extraction technique where shades of grey and augmentation takes place in the processed images. Usage of R2U-net for segmentation and using EfficientNet ,SENet -154 and InceptionNet to classify the skin lesion. Efficient is used for scaling and uniform scaling in all dimensions including depth, width and resolution. SENet-154 is a squeeze and excitation block which enables the network to perform a dynamic channel wise feature recalibration.Inception V3 archives an accuracy in top5 of ImageNet and reduces the computational cost without any decrease in speed and accuracy.

Ahmad Naeem ,et al,(2020) preprocess the dataand is ensemble with a dense layer, hence combining all the models with ensemble.Median method is applied on the outlier or on the missing value. For binary features, label encoding is performed and for non binary, one-hot encoding was used. These models with ensemble we fused together by achieving a sensitivity of $81.5 \%$ and specificity of $97.7 \%$ [5].

Later, the work was done on the classification of skin lesion images using U-net algorithm .U-net algorithm is developed for image segmentation in medical image analysis that can precisely segment images using a limited amount of training data. In this study, they $[2,4,10]$ detected skin lesions based on the U-net algorithm.

FredericoGuth and Teofilo E. deCampos (2018)defines about the semantic segmentation based on medical images. It consists of a dataset for ISIC 2019 dataset where it demonstrates the robustness of the proposed model in image segmentation task. The input images contain 
two spots one is a bright spot and the other which is not a target. The non target lesion will be brighter than the targeted lesion. The U-net model segments the region of interest accurately [2].

It also uses VGG-16 and Inception V3 models for skin cancer segmentation to check the accuracy. [2] also performs segmentation in other medical images such as retinal blood vessel segmentation and lung related disease where U-net is used to segment the images present in the database.

Md ZahangirAlom, et al, uses U-net 34 model to segment the biomedical images where the output of the images is the same dimension as the input images [4] . A u-net takes in the data and learn the contextual information based on the convolutional network. It fine tunes the network in a higher resolution data to learn fine details. The training of the model is with $128 \times 128$ resolution images used for the training phase. It first freezes the first layer and then defines the optimal learning by implementing the one batch to be trained with different learning rates. The training phase obtains a convergence of only 30 epochs and then it unfreezes the model by only keeping the batch normalization layer frozen.

There are 2 strategies followed, BestDice and Ensemble where BestDice is used to predict the input on the basis of best dice index on the validation phase of the training set which is a coefficient which usually ranges from 0 to 1 . Ensemble is a 3 fold of the training set which is trained with the BestSice index . The data augmentation is done on the ISIC 2018 dataset which comprises 2597 training images. The best result is obtained using an Ensemble strategy[4] which achieves $85.3 \%$ Jaccard index and $78.4 \%$ of threshold Jaccard index. The BestDice strategy has an online score with validation set of $75.5 \%$.

Hemanth Nadipineni ,(2020) uses ISIC 2019 dataset which is given for preprocessing where certain images of the lesions are surrounded by the black color. So the region which is unwanted is marked with a pixel value as 0 therefore, removing the darker part of the images. It helps to concentrate more on the region of interest and to decrease the size of the image lessens the computational time [10].

The complete dataset for training is segregated into 5 folds where each fold has an image related to one of the two classes such as random sized cropping and same sized cropping. Large ensemble out of all trained models is created with an optimal subset .It is performed in CNN one path and CNN dual path. The CNN one path takes in the images which is formed by multiplication of the original images and its segmented mask is given as input to the dual path which takes both the original images and the segmented images as the input.

The accuracy of CNN one path is less than the CNN dual path as the number of neuron differences on both the models. There was an improvement in accuracy by training where the images are multiplied with the segmented mask and keeping the unwanted region pixel value as 0 .

U-net architectureis used for the segmentation mostly developed for medical images. The model is made robust by using $\mathrm{K}$ fold cross validation algorithm. The model with each fold is given for 10 times training to act as the validation set which has an increase in the accuracy when data augmentation was involved.

The major work was carried out on developing models based on CNN variants such as ResNet and VGG19 for classifying skin lesions using dermoscopy images [6].The dermoscopy images are preprocessed to remove the unwanted regions by removing the dark circles in the images around the lesion region, contrast balancing, etc. ResNet is increasing network depth, it avoids negative outcomes. So, it is easier to increase the depth which has fast training and higher accuracy. 
The extracted features from the convolutional layer are given to the classifier to analyze the performance and to differentiate the different types of skin lesions. The ResNet 50 model classifies the skin lesion from the ISIC repository consisting of 1800 images of benign moles and 1497 images of malignant moles for the lesion prediction.

G.s. Jayalakshmi, Sathiesh Kumar, (2019) performs image normalization by dividing all the RGB values to 255 . Features of the images are extracted and down-sampled using convolutional and max pooling layers . The flatten layer is utilized for remodeling the feature maps to identity vectors. The input images are classified into benign and malignant based on their pixel value [6].

The skin cancer dataset evaluation achieves a model accuracy and model loss by identifying various sets of images with different epochs. It has a step loss of 0.1494 , validation loss of 0.4231 and validation accuracy of 0.820 obtaining the classification accuracy for the fifty epochs is $94.8 \%$. The skin is classified by adding additional training to the model on 100 samples and validating those models on 26 samples. It leads to sample loss of 0.44 and a step loss of 0.123 by obtaining a validation accuracy of 0.443 and the classification accuracy of 15 epochs is $90.7 \%$ using a ResNet-50 model layer.

Skin cancer classification is also performed by training on 5636 samples and validating the model for 149 samples.It has a step loss of 0.077 and a validation loss of 0.480 , validation accuracy of 0.865 and obtains classification accuracy for 20 epochs is $97.32 \%$ on VGG-16 model.

The classification is done using AlexNet, which is a fully connected layer which is replaced by the convolution layer, and is performed by training the model for 1300 image samples. Validation of the model is done on 149 samples which provides a step loss of 0.223 , validation loss of 8.710 and the validation accuracy of 0.444 and obtains a classification accuracy for 15 epochs is $81.8 \%$ on AlexNet.

Skin cancer classification using a GoogleNet Inception v3 model is performed by training the model on 3374 image samples. Feature extraction is done which provides a step loss of 0.088 , validation loss of 0.444 and validation accuracy of 0.8523 and obtaining a classification accuracy for 15 epochs is $96 \%$ on GoogleNet Inception v3 model. As the sample is increased the deep learning algorithm provides better accuracy and by avoiding overfitting which will degrade the accuracy on test images.

Mohamed A. Kassem, et al,(2021) have detected the skin lesion using certain pedagogical activity that dissects the procedures to train the convolutional neural network on the ISIC dataset containing images of skin lesions associated with eight different skin cancer categories where the activity was made available open-source and its execution does not require the installation of software [1] .

ISIC 2019 dataset were used where these images are detected using GoogleNet and multi class SVM. GoogleNet consists of 22 layers which is formed by the researchers. The multi class SVM was used to handle the imbalance of classes of images in dataset where it is used by replacing the sampling based on the imbalanced class data.SVM classify the new images present in the test data where it classifies and calculates the similarity between each classes of the dataset where $80 \%$ of the dataset is taken as training dataset .

The outlier images were detected by applying it on the unknown images by applying certain rules. If the score is less than 0.5 for 8 classes will be considered as unknown. Detection of unknown classes cannot be trained and tested on the same device and hence need different hardware specifications.

The major work for developing a CNN model with a SVM classifier [7] to classify the dermoscopy images in HAM1000 dataset. The training database is smaller and a limited 
number of tests were conducted. For better differentiation between different types of skin lesions in terms of appearance andfeatures are classified using dermoscopy.

Qilin Sun ,et al,(2021)classifies the skin lesion based on two dataset ISIC 2019 and PH2 dataset. The images are resized and the shade of gray color is applied and then the color gain in the RGB channel is recorded [3] . The patient's additional information is encoded by onehot encoding techniques. Usage of EfficientNet has been pre trained on the ImageNet dataset which is a large visual database designed for visual object recognition.

It consists of 8 different types of model which follows scaling rules for the adjustment for larger images. It incorporates the additional information of the patients and fuses it with the convolutional neural networks.EfficientNEt improves the accuracy by uniform scaling of all the dimensions such as depth, width and the resolution of the images using a compound coefficient.

The data augmentationin the training model is done both geometrically and pixelwise with gaussian blur and gaussian noise. The scaling and shifting properties of the geometric augmentation are later used as meta features where 60 epochs are been trained with a batch size of 16 . It shows a result with a balanced multiclass accuracy of $88.8 \%$ on a single model and $89.5 \%$ on embedding the solutions. It has obtained a low computational complexity.

AmirrezaMahbod, et al, (2019) uses AlexNet and VGG16 which is a variant of VGGNet which is used as a feature extractor. VGG16 is used to prevent the overfitting of a number of training images [7]. The features are used to train the non linear support vector machine (SVM) classifier where different classifiers are trained and then the results are fused together which is used as the result for classification.

The evaluation is done using a ISIC 2017 dataset where the validation set consists of 30 of malignant melanoma, 42 of seborrheic keratosis and 78 of benign nevus dermoscopy images. The raw images were resized for AlexNet and VGG16. The results were obtained by the average of outputs obtained over five iterations.VGG16 consist of 16 layers with five blocks with max pooling layers. AlexNetha a probability distribution over 1000 classes.

The system is performed on the limited dataset and using shallower network for better results. Usage of DenseNet can lead to better results and by resizing the images have benefited the image classification using different methods.

Halil Murat Ünver and Enes Ayan,(2020)uses a divide and conquer technique to divide n-ary classification of the ISIC 2019 dataset which converts it into smaller problems making the problem easier. Data normalization which is an automatic preprocessing is done before giving it to classification models [9] .

Data augmentation is done including resizes, rotation ,noises and distortions and cuts. all the validated images given for testing are resized to $380 \times 380$. The sampling technique is used for multiplying the image data of different classes.

Recent convolutional neural networks are used [9] for imbalanced data and over-fitting. The over-fitting was overcomed by combination of different methods by modifying the models and training the model from the beginning without using the pretrained model. For imbalance of the dataset different standard up sampling techniques were used.

Using the asymmetric focal binary loss function helped to overcome the data imbalance where the asymmetry parameter depends on the level of data imbalance. The dataset used for[9] is ISIC 2019 and HAM1000 dataset.

Roberta B. Oliveiraa ,et al,(2018) performs a review of computational methods for classification of the pigmented skin lesion images. Feature is extracted from both dermoscopic and macroscopic images [11]. The images are analysed using ABCDE rule based on 
asymmetry, border,color and diameter where the features are extracted and automatic diagnosis of skin lesion .

The pattern analysis is done by examining the size and distribution based on the pattern visible in dermoscopy images which is divided into local and global patterns. Global patterns are textural structures and local patterns are the irregular or regular structure to define whether a skin lesion is malignant or benign. The shape features the lesions is a border irregularity which is examined by dividing the region into two sub regions. Geometrical measures from segmented lesion regions are computed to assess the border irregularity.

The color variation [11] ,RGB color space is used for skin lesion representation. The standard deviation, skewness and variance is computed for each color channel for the lesion regions. Textural analysis is used to assist in discriminating between malignant and benign skin lesions. The features are also extracted based on histograms based on the intensity and gradient histogram to represent the texture features.

Features are selected based on pattern analysis and skin lesion classification to select only the important features and removes the irrelevant features to maintain the dimensionality which helps the process to reduce the feature extraction time reduces complexity and improves the classification accuracy rate. Several search algorithm such as incremental stepwise and random have been used for the feature selection process. The classification takes the details based on the feature extracted from the skin lesion images. The classification occurs by dividing the image samples from the training set and the test set. Feature normalization is applied to solve the problems occurring in the different ranges where $\mathrm{z}$ score normalization is done for data normalization. The unbalanced dataset is handled by overcomingover-fitting and under-sampling and the classification method is based on the support vector machine and other ensemble methods to discriminate the skin lesion images. The k-fold cross validation and leave-one-out is a cross validation procedure to classify the skin lesion. The computational methods based on the issues to perform more effectively by using the evaluation of new classifiers to improve the result and increase the accuracy.

Use of deep residual network [11] to overcome the issues based on lower exactness by making a plain neural network with a more profound layer. Pre-processing of data is done to improve the picture differentiation by applying shading or alignment or expelling picture relics. The dermoscopy images are resized to capture the wanted information from region of interest and perform a segmentation .

A filter examines the couple of pixels after making a component map predicting the class of each element . Max-pooling layers measure the data in the convolutional layer by keeping the significant data.Dropouts is a regularization technique which closes off various arbitrary neurons at predefined layer. Data augmentation overcomes the overfitting in the model. The ISIC 2019 is given as the training data with the ground reality document. It obtains an accuracy of $92 \%$ and the approval exactness shows the information which is anticipated accurately by the model.

Yuexiang Li and Linlin Shen,(2018) compares the performance of the network with different depths and the results caused by adding different components such as batch normalization. Residual network along with the improved version of FCRN-88 achieves a good result in segmentation of the network. Lesion Indexing Network is constructed for skin cancer analysis. The dataset is trained using different augmentation techniques [13] .

In the testing stage[13], accepts the input images with different sizes which resizes the image into two scales. The different scales sum up to yield a rough probability matrix. Lesion index calculation unit takes in the rough probability matrix and is normalized to $[0,1]$. Refined 
takes in the average possibility by getting the different indexes with different classes of the skin lesion.

The dermoscopic image features are extracted using ISIC 2017 . The extracted dataset is imbalanced. The patches consist of only background images where data augmentation balances the number of images in different classes. Thos augmented images are sent for training the lesion feature network where it has 12 convolutional layers for feature extraction. The 12 layers are separated into 4 stages .

Average pooling and max pooling techniques are used, the network is trained with the softmax loss. The weight is assigned to a different training dataset to calculate the softmax loss and the proposed Lesion Feature Network is developed using Keras.The proposed LFN obtains an average precision of 0.422 and sensitivity of 0.693 for the dermoscopy image feature extraction.

DevanshBisla ,2019) uses data purification of data to remove the occlusions and also removes the up-sampling blocks using a U-net architecture, further the segmentation model is passed through the binary filling procedure to fill up the empty holes present in the segmented mask [13] .The segmentation is performed in the ISIC 2017 dataset. The accuracy obtained for classifying the images based on three classes is computed based on a confusion matrix .

S.Ravi and MuralikrishnaPuttangunta (2021) performsa study on deep learning techniques and its history since 1940 and how it emerged in medical applications .It shows how medical images are classified using deep learning techniques where several supervised and unsupervised techniques were discussed based on recurrent ,auto encoder and convolutional neural networks [15] . Various techniques were reviewed for image classification and segmentation. CNN and its variants identifies and categorizes the nodules in the input images with a better result including TensorFlow ,Pytorch and Theano are discussed. 


\section{System Design}

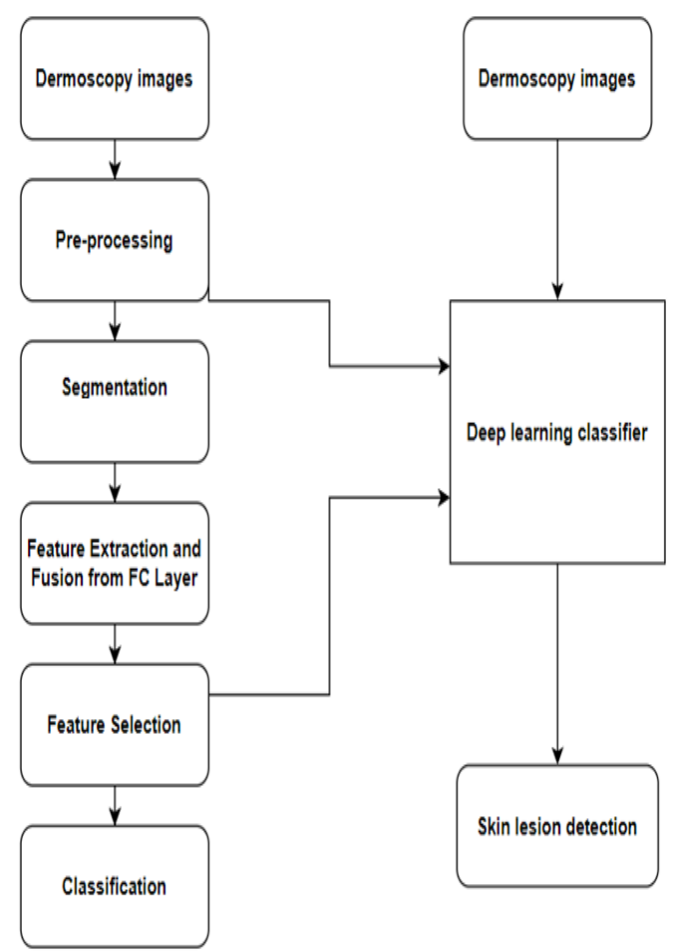

The dermoscopy images is given for pre-processing where the sizes can be changed without any changes in the resolution of the skin lesion images The preprocessing resizes the images for feature detection without any changes the shape of the skin lesion. After image preprocessing it is then given for image segmentation using a U-Net algorithm.

A. U-Net Algorithm

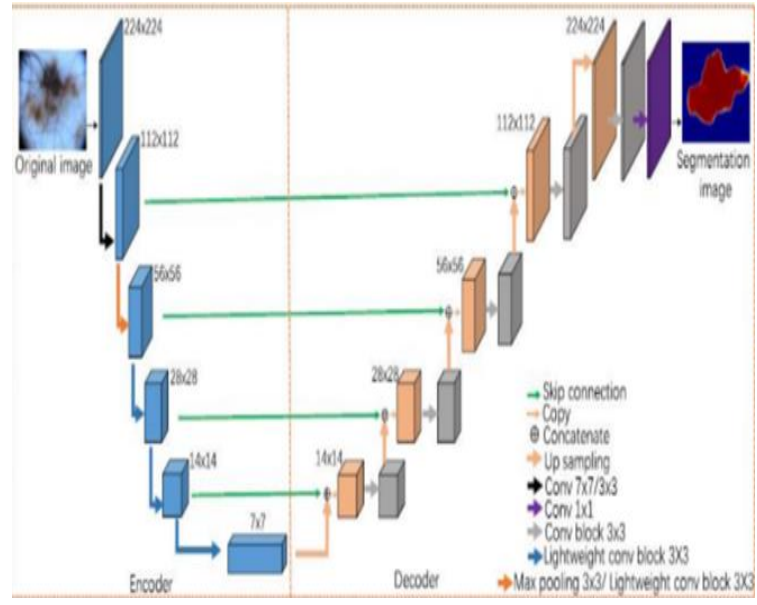


U-net is a convolutional network which is developed for the segmentation of biomedical images. U-net is the expanded version of CNN with few changes in the architecture.It localizes the objects in the image. It takes in the original images and then converts them into the ground truth binary mask and then it overlays with the original images.

U-net comprises two parts:encoder and decoder. Encoder captures the context via feature map where feature map is an output of one filter layer applied to the previous layer. Decoder allows precise localisation to retain the spatial information for all down sampling and max pooling in the encoder stage.

The original dataset is divided into two groups for testing and training. The original images are in RGB format which are converted into binary masks which are in black and white. The unwanted region is given as 0 and the region of interest is given as 1 .

\section{B. ResNet-50}

ResNet can extract the features from a very large dataset and as incremental learning to train the classifier for the extracted features and are used to solve the problem by vanishing gradients.

In ResNet it uses a skip connection which skips the connection from training a few layers and connects directly to the output.

Instead of learning underlying layer mapping, the network also learns to get in the residual mapping. The main advantage of skip connection is because if any layer performance is hurt then it will be skipped using regularization. So the result is training the neural network architecture without any vanishing gradient problem.ResNet improves the efficiency by minimizing the percentage error.

ResNet 50 is a convolutional network which has 50 neural network layers. It modifies the bottleneck design due to concern for time taken to train the model by using a stack of 3 layers instead of 2 layers. Each 2 block layer in ResNet-34 is replaced with 3 layer block forming the ResNet50 architecture. Keras is a deep learning API which comes with several pretrained models including ResNet50.

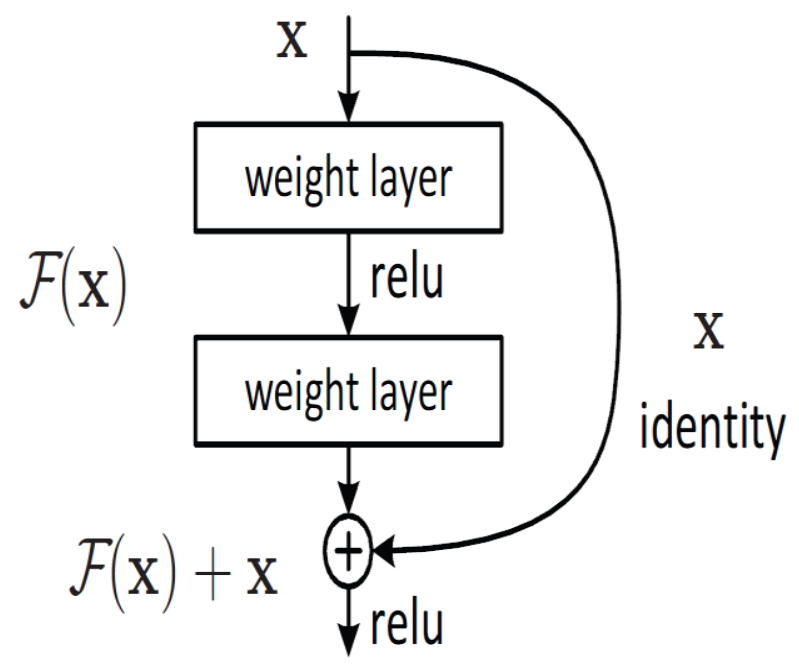


As the network depth increases the accuracy starts getting saturated and degradation takes place. To overcome this problem Residual learning framework is used. Every stacked layer fits the desired underlying mapping and fits with the residual mapping. The formulation of $\mathrm{F}(\mathrm{x})+\mathrm{x}$ is used for feed forwarding the neural networks by using shortcut connections by skipping the connection for one or more layers. These shortcut connections perform identity mapping to add the output to their stacked layers.

ResNet is easy to optimize and can gain the accuracy easily from increased depths producing better results than the previous networks.

\section{Conclusion}

This review paper discusses the various deep learning techniques for skin lesion detection and classification to develop an accurate skin lesion detection system. The paper shows that technology has evolved a lotover these years for skin lesion detection. The improvements on performance in the areas of image preprocessing, image segmentation, feature extraction and classificationcan be seen in this paper.This review is focusedon the papers related toU-net algorithm for image segmentation and comparing the different variants of CNN where ResNet has achieved a better accuracy compared to other variants for feature extraction and classification.

\section{References}

[1] Mohamed A. Kassem, Khalid M. Hosny And Mohamed M. Fouad "Convolutional Neural Network for Skin Lesion Classification: Understanding the Fundamentals Through Hands-On Learning”, Mar 2021

[2] FredericoGuth and Teofilo E. deCampos "Skin lesion segmentation using a U-Net and good training strategies", Nov 2018

[3] Qilin Sun ,Chao Huang, Minjie Chen, Hui Xu and Yali Yang "Skin Lesion Classification Using Additional Patient Information”,Apr 2021

[4] Md ZahangirAlom,Mahmudu Hasan , Chris Yakopcic,Tarek M. Taha and Vijayan K. Asari "Recurrent Residual Convolutional Neural Network based on U-Net (R2U-Net) for Medical Image Segmentation", Feb 2018

[5] Ahmad Naeem, Muhammad Shoaib Farooq ,Adel Khelifi ,Adnan Abid "Malignant Melanoma Classification Using Deep Learning: Datasets, Performance Measurements, Challenges and Opportunities", Jun 2020

[6] G.s. Jayalakshmi, Sathiesh Kumar "Deep neural networks approach to skin lesions classification A comparative analysis",Feb 2019

[7] AmirrezaMahbod, R. Ecker, I. Ellinger "Skin Lesion Classification Using Hybrid Deep Neural Networks",May 2019

[8] Ali Madooei, Mark S. Drew, Maryam Sadeghi, and M. Stella Atkins "Pre-processing Algorithms for Melanoma Image Enhancement”, Dec 2018

[9] Halil Murat Ünver and Enes Ayan "Skin Lesion Segmentation in Dermoscopic Images", Mar 2020

[10] Hemanth Nadipineni"Method to classify skin lesions using dermoscopic images", Aug 2020

[11] Roberta B. Oliveiraa, João P. Papab , Aledir S. Pereirac and João Manuel R. S. Tavares "Computational Methods for Pigmented Skin Lesion Classification in Images: Review and Future Trend", 2018

[12] Niharika Gouda and Amudha J "Skin Cancer Classification Using ResNet" Jun 2020

[13] Yuexiang Li and Linlin Shen "Skin Lesion Analysis towards Melanoma Detection Using Deep Learning Network", Feb 2018. 
[14] DevanshBisla, Anna Choromanska, Russell S. Berman Jennifer A. Stein, David Polsky "Towards Automated Melanoma Detection with Deep Learning: Data Purification and Augmentation”, Jun 2019

[15] S.Ravi and MuralikrishnaPuttangunta "Medical image analysis based on deep learning approaches", Apr 2021 